\title{
Text Entry Performance Evaluation of Haptic Soft QWERTY Keyboard on a Tablet Device
}

\author{
Byung-Kil Han ${ }^{1,2}$, Kwangtaek Kim ${ }^{2,3}$, Koji Yatani ${ }^{2}$, and Hong Z. Tan ${ }^{2}$ \\ ${ }^{1}$ Korea Advanced Institute of Science and Technology, Daejeon, Republic of Korea \\ ${ }^{2}$ Microsoft Research Asia, Beijing, P.R. China \\ ${ }^{3}$ Yonsei University, Seoul, Korea \\ hanbk@robot.kaist.ac.kr, \\ kwangtaekkim@yonsei.ac.kr, \\ $\{$ koji, hongtan\}@microsoft.com
}

\begin{abstract}
.
Recently touch screens are widely used for mobile devices to provide intuitive and natural interactions with fingertips. However, the lack of tactile feedback makes it difficult for users to receive key-click confirmation during text entry on soft keyboards. This paper examines the effect of tactile feedback on typing performance with the soft QWERTY keyboard: the most commonly used multifinger text entry method on tablet devices. We implemented tactile feedback hardware and software to simulate the key-click effect on a commerciallyavailable mobile tablet (Microsoft Surface Pro). We conducted a typing experiment to measure user performance and preference. The participants transcribed given phrases under three sensory feedback conditions: visual only, visual and audio, and visual and tactile. The results are unexpected; we did not find any significant difference in terms of typing performance, and user preference was as positive as the audio condition though better received than the visual only condition. This study thus reports different findings from previous work studying text entry on handheld devices, encouraging further examinations to fully understand the effect of tactile feedback on text entry in tablet devices.
\end{abstract}

\section{Keywords: Haptic I/O, HCI, Mobile Device}

\section{Introduction}

Touch screens are widely used for mobile phones and tablet devices. Touch-sensitive screens reduce physical hardware for input and create various forms of virtual buttons displayed on the screen. Therefore, interactions with these devices become flexible and intuitive. However, removing the physical buttons leaves insufficient tactile experience during interactions. For typing on the soft keyboard, the lack of tactile responses prevents users from perceiving key-click confirmation and causes slower and less accurate typing. Although almost all mobile and tablet devices provide key

adfa, p. 1, 2011.

(C) Springer-Verlag Berlin Heidelberg 2011 
click sound feedback for a key press, such audio feedback may not be appropriate in some situations such as noisy places or quiet places like theaters. In addition, tactile sensation in the fingertips helps with the localization of finger in the air that contributes to more accurate typing performance [1]. Users also tend to press and pause while typing on the touch screen because they do not receive key-click confirmation [2]. Therefore, many researchers consider haptic feedback as playing a central role in touch-screen-based typing tasks.

Brewster et al. [3] studied the effects of tactile feedback on soft keyboard typing using a stylus on PDAs. For tactile feedback, they attached a commercial tactor to the backside of the device. The results of their experiment showed that, compared with the soft keyboard without tactile feedback, tactile feedback helps to improve typing speed and accuracy. They also indicated that tactile feedback should also be helpful for typing with fingertips, a prediction that was confirmed later by Hoggan et al. [4]. Hoggan et al. tested two kinds of tactile feedback: vibrating the entire touch panel using the device's built-in tactor, and generating tactile feedback by selectively turning on and off multiple actuators attached to the device to simulate localized feedback for each key. They found that tactile feedback improved typing performance, which was further improved upon by simulated localized feedback.

McAdam et al. [5] investigated the effect of tactile feedback on text entry with tabletop computers. They attached tactile actuators to the user's body, and provided tactile feedback synchronized with text entry. They found that tactile feedback helped to improve the typing speed.

More recently, Kim and Tan [6] instrumented a flat keyboard without moving keys and studied touch typing performance using various key-click confirmation methods including visual, auditory, local and global haptic feedback. Their results show a trend that local haptic keyclick feedback led to the highest typing speed, followed by global haptic feedback and auditory feedback.

The prior work motivated us to investigate the effect of tactile feedback on typing with tablet devices that have recently demonstrated a very high market penetration rate. The present study focuses on multi-finger text entry using a soft QWERTY keyboard on a touchscreen as it is the most commonly-used input method for tablets. Although prior work suggests positive effect of tactile feedback on phones, PDAs and flat keyboards, few studies have quantified typing performance gain due to tactile feedback using multiple fingers and on a software-based keyboard on a piece of glass. Our contribution therefore lies in the quantitative assessment of user performance and preference of tactile feedback on soft QWERTY keyboards in tablets. We utilized Microsoft Surface PRO as a platform, and developed a keyboard with tactile feedback providing realistic key click effect. Using this platform, we conducted an experiment to compare typing performance under different sensory feedback conditions. Although our results confirm the positive effect of haptic feedback on typing performance, the differences from the audio feedback condition were not as apparent as reported in previous work studying in handheld devices. This study thus calls for further investigations on how tactile feedback on text entry could impact in tablet devices. 


\section{Methods}

\subsection{Apparatus}

We embedded multiple piezoelectric actuators under the touch screen of a Microsoft Surface PRO to create key-click sensation on a soft QWERTY keyboard. The actuators were driven by custom-designed piezo-driver circuits that amplified the input voltages to piezos up to $200 \mathrm{Vpp}$ (Figure 1).

The typing tasks involved transcribing phrases using the tablet. We chose the phrases from the set created by Mackenzie and Soukoreff [8], and randomly divided them into fifteen-phrase blocks. The phrases were displayed at the top of the touch screen, and remained visible until the typing ended (see Figure 1). A text box under the phrase showed the user's entry. The entries in the text box were perfectly aligned with the given phrase above it so that the user could quickly glance and compare the two lines to decide whether the phrase had been typed correctly.
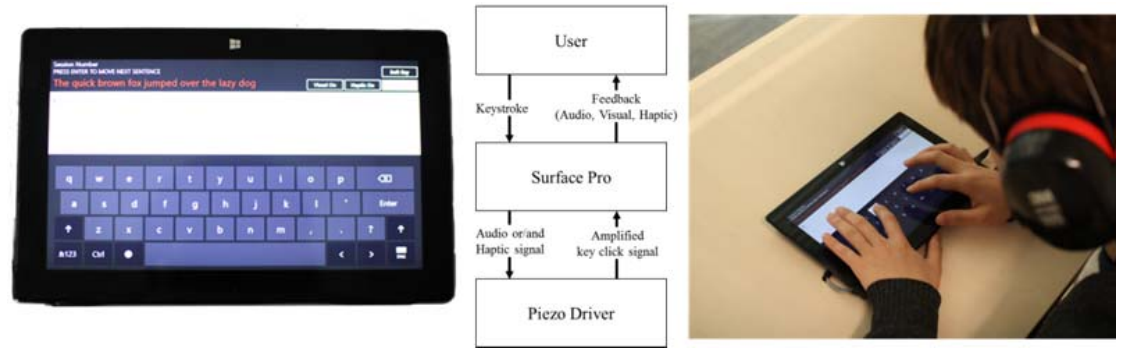

Fig. 1. Our keyboard prototype (left) and control diagram (center). Visual feedback was provided by displaying the typed letter and highlighting the pressed button (right).

\section{$2.2 \quad$ Feedback Stimuli}

Our hardware was synchronized with key presses to generate visual, aural and tactile feedback with $11.5 \mathrm{~ms}$ delay, primarily caused by Windows operating system, the soft keyboard application and the piezo driver circuit. The visual feedback highlights the pressed key by reversing the color scheme and shows the typed key in the text box (Figure 1). The audio feedback used the built-in key-click sound in the device, which was a combination of several sinusoidal functions with a fundamental frequency of $600 \mathrm{~Hz}$. The sound intensity was set to $52.2 \mathrm{dBA}$, as measured through a headset. The haptic feedback was modified from the key-click feedback signals reported by Chen et al. [7], and contained two- cycles of a $500 \mathrm{~Hz}$ square wave. The peak acceleration of the tactile key click feedback was measured to be in the range 2 to $3.5 \mathrm{~g}$ (with $2 \mathrm{~g}$ at the center and $3.5 \mathrm{~g}$ at the bezel of the touchscreen shown in Figure 1). 


\subsection{Conditions}

Three feedback conditions were used: visual feedback only (V), visual and aural feedback (VA), and visual and tactile feedback (VH). The visual feedback was always available as this is the default setting for any soft QWERTY keyboards. This design allowed us to examine the effect of the additional auditory or tactile feedback by comparing the results from the $\mathrm{VA}$ and $\mathrm{VH}$ conditions against the $\mathrm{V}$ condition. The order of the conditions across the participants was randomized.

\section{$2.4 \quad$ Procedures}

The entire experiment was divided into three sessions. Each of the sessions included five fifteen-phrase blocks under one of the three conditions. Before starting each session, participants were given time to sufficiently get familiarized with the device and the conditions. For each phrase, they were asked to read the given phrase first until they became familiar with it, or memorize it before starting to type. They then transcribed it as quickly and as accurately as possible [9]. To prevent fatigue and loss of concentration, participants were instructed to take a rest of about three to five minutes after each block.

The participants were asked to sit in front of a desk where the tablet was placed to transcribe phrases for training and the test. During the test, the participants were asked to wear earplugs during the $\mathrm{V}$ and $\mathrm{VH}$ conditions to block noises from the apparatus and a headset during the VA condition for receiving audio feedback.

After finishing all the typing, the participants responded to our questionnaire about their experience and preferences for the different sensory feedback. Each statement was composed of the following 7-point Likert-scale questions: [Pleasure] This feedback was pleasant to use; [Comfort] This feedback was comfortable to use; [Physicality] This feedback felt like pressing a real button; [Confirmation] I always felt the device receive my key press; [Frustration] I felt frustrated under this feedback condition; [Error reduction] This feedback condition helped me avoid errors; and [Typing speed] This feedback condition helped me type quickly.

\subsection{Participants}

Eleven participants from age 22 to 27 years old (P1-P11; 8 males; 10 right handed; average age 24.42 years old, std. 1.68 years old) took part in the experiment. All participants claimed to be skilled typists, and to have at least one year of experience using touch screen devices.

\subsection{Data Analysis}

The typing performance metrics developed by Soukoreff et al. [9-10] were used in the present study. We used keystroke per character (KSPC) for assessing typing efficiency (lower is better), words per minute (WPM) for assessing typing speed (higher is better), and corrected error rates (CER) and uncorrected error rates (UER) 
for assessing typing accuracy (lower is better). We removed outliers which were beyond $2 \mathrm{SD}$ from the mean per participant, condition, and block before the analysis.

\subsection{Typing Performance}

Figure 2 summarizes the typing performance in our experiment. We ran a repeatedmeasure ANOVA test after conducting Mauchly's sphericity test. We did not observe any case of sphericity violation, and we thus report the unadjusted results of ANOVA tests. The WPM ranged from 27.3 to 29.2, with VA appearing to have the highest typing speed. An ANOVA on the feedback conditions found no significant differences among the WPMs $\left(F_{2,22}=1.32, p=.29\right.$, partial $\left.\eta^{2}=.12\right)$. Similarly, no significant difference was found in $\operatorname{KSPC}\left(F_{2,22}=0.75, p=.49\right.$, partial $\left.\eta^{2}=.07\right)$. The average KSPC values were $1.25,1.24$ and 1.28 for the $\mathrm{V}$, VA and $\mathrm{VH}$ conditions, respectively.

Regarding accuracy, the average CERs were 10.3, 9.7 and 11.1 for V, VA and VH conditions, respectively. The results from the ANOVA test did not showed a significant effect of feedback condition $\left(F_{2,22}=0.94, p=.41\right.$, partial $\left.\eta^{2}=.09\right)$. The average UERs were $0.57,0.43$ and 0.47 for the $\mathrm{V}$, VA and $\mathrm{VH}$ conditions, respectively. The ANOVA test did not show the feedback condition to be a significant factor $\left(F_{2,22}=2.06, p=.15\right.$, partial $\left.\eta^{2}=.17\right)$.

Overall, our results did not show any difference in any of the performance metrics due to feedback conditions. The following subsection shows how user experience was influenced by the feedback conditions through the questionnaire results.
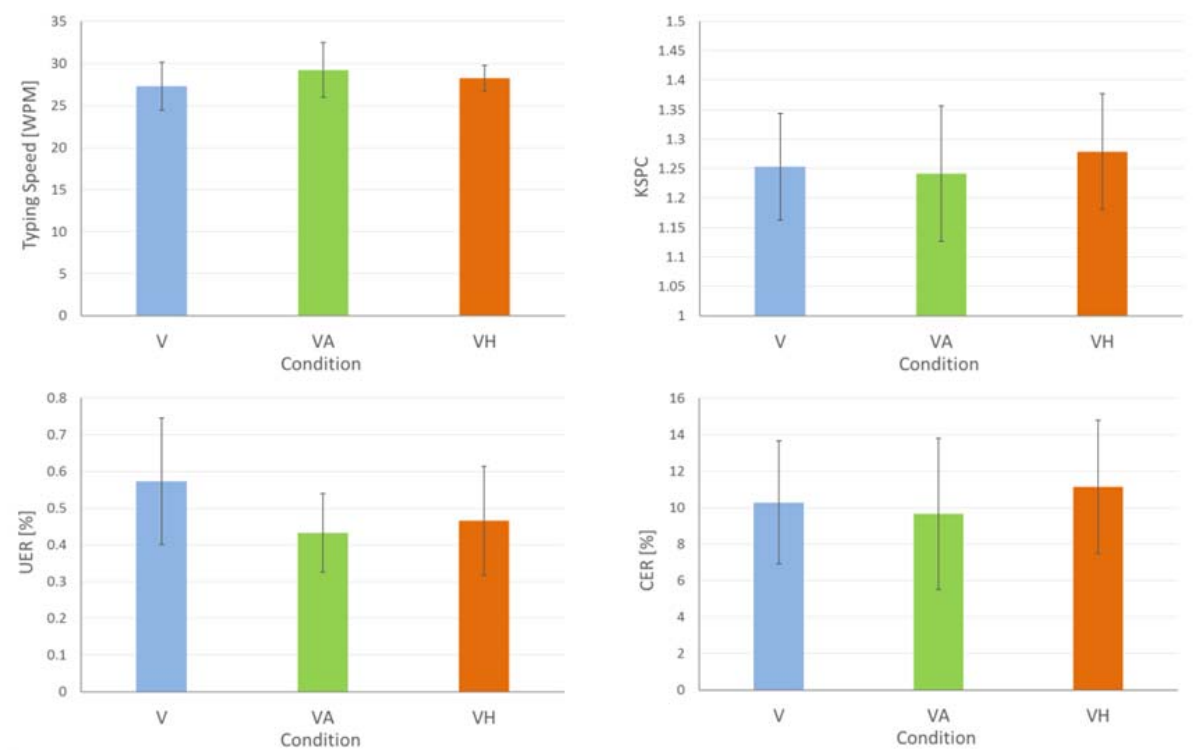

Fig. 2. Typing performance results. The error bars represent $95 \%$ confidence intervals. Except for the typing speed, a lower number means better performance. 


\subsection{User Preference}

Figure 3 and Table 1 summarize the results from the questionnaire. As seen in Table 1 , we observed significant differences between the $\mathrm{V}$ condition and the VA and $\mathrm{VH}$ conditions. This indicates that the additional auditory or tactile feedback was perceived to be helpful in increasing the comfort level. However, contrary to what was expected from prior work, we did not observe any significant difference between the VA and VH conditions. Our study found a significant difference in the Physical statement between the $\mathrm{V}$ and $\mathrm{VH}$ conditions, but not between the $\mathrm{V}$ and VA conditions. Nevertheless, the difference between the VA and VH conditions was not found to be significant. Our study therefore concluded that the differences between the additional audio and tactile feedback were not as apparent as those reported by previous work.

Table 1. Nonparametric test results of our questionnaire ratings.

\begin{tabular}{|c|c|c|c|}
\hline & $\mathrm{V}-\mathrm{VA}$ & $\mathrm{V}-\mathrm{VH}$ & $\mathrm{VA}-\mathrm{VH}$ \\
\hline Pleasure & $\begin{array}{l}\text { Significant } \\
(\mathrm{Z}=2.971, \mathrm{p}<.05, \mathrm{r}=0.858)\end{array}$ & $\begin{array}{l}\text { Significant } \\
(\mathrm{Z}=3.104, \mathrm{p}<.05, \mathrm{r}=0.896)\end{array}$ & $\begin{array}{l}\text { Non-significant } \\
(\mathrm{Z}=1.930, \mathrm{p}=.162, \mathrm{r}=0.557)\end{array}$ \\
\hline Comfort & $\begin{array}{l}\text { Significant } \\
(\mathrm{Z}=2.754, \mathrm{p}<.05, \mathrm{r}=0.795)\end{array}$ & $\begin{array}{l}\text { Significant } \\
(\mathrm{Z}=2.831, \mathrm{p}<.05, \mathrm{r}=0.817)\end{array}$ & $\begin{array}{l}\text { Non-significant } \\
(\mathrm{Z}=1.933, \mathrm{p}=.159, \mathrm{r}=.0558)\end{array}$ \\
\hline Physicality & $\begin{array}{l}\text { Non-significant } \\
(\mathrm{Z}=2.060, \mathrm{p}=.12, \mathrm{r}=0.595)\end{array}$ & $\begin{array}{l}\text { Significant } \\
(\mathrm{Z}=2.821, \mathrm{p}<.05, \mathrm{r}=0.814)\end{array}$ & $\begin{array}{l}\text { Non-significant } \\
(\mathrm{Z}=2.356, \mathrm{p}=.054, \mathrm{r}=0.681)\end{array}$ \\
\hline Confirmation & $\begin{array}{l}\text { Significant } \\
(\mathrm{Z}=2.952, \mathrm{p}<.05, \mathrm{r}=0.852)\end{array}$ & $\begin{array}{l}\text { Significant } \\
(\mathrm{Z}=2.944, \mathrm{p}<.05, \mathrm{r}=0.850)\end{array}$ & $\begin{array}{l}\text { Non-significant } \\
(\mathrm{Z}=1.807, \mathrm{p}=.213, \mathrm{r}=0.522)\end{array}$ \\
\hline Frustration & $\begin{array}{l}\text { Significant } \\
(\mathrm{Z}=2.323, \mathrm{p}=06, \mathrm{r}=0.670)\end{array}$ & $\begin{array}{l}\text { Significant } \\
(\mathrm{Z}=-2.226, \mathrm{p}=.06, \mathrm{r}=0.643)\end{array}$ & $\begin{array}{l}\text { Non-significant } \\
(\mathrm{Z}=1.414, \mathrm{p}=.471, \mathrm{r}=0.408)\end{array}$ \\
\hline Error reduction & $\begin{array}{l}\text { Significant } \\
(\mathrm{Z}=2.539, \mathrm{p}<.05, \mathrm{r}=0.733)\end{array}$ & $\begin{array}{l}\text { Significant } \\
(\mathrm{Z}=-2.992, \mathrm{p}<.05, \mathrm{r}=0.864)\end{array}$ & $\begin{array}{l}\text { Non-significant } \\
(\mathrm{Z}=2.126, \mathrm{p}=.099, \mathrm{r}=0.614)\end{array}$ \\
\hline Typing speed & $\begin{array}{l}\text { Significant } \\
(\mathrm{Z}=2.716, \mathrm{p}<.05, \mathrm{r}=0.784)\end{array}$ & $\begin{array}{l}\text { Significant } \\
(\mathrm{Z}=2.555, \mathrm{p}<.05, \mathrm{r}=0.738)\end{array}$ & $\begin{array}{l}\text { Non-significant } \\
(\mathrm{Z}=0.351, \mathrm{p}=.725, \mathrm{r}=0.101)\end{array}$ \\
\hline
\end{tabular}

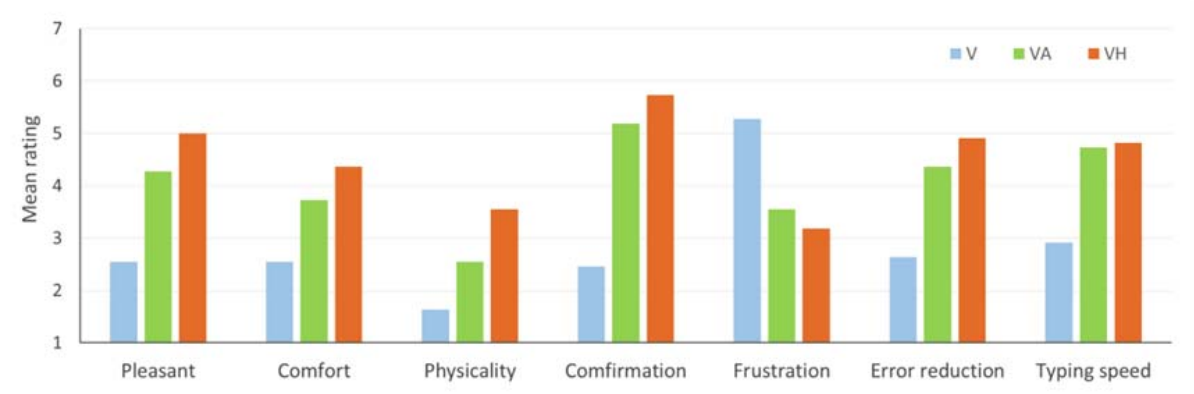

Fig. 3. Results of the post-experimental questionnaire.

\section{Conclusion}

The present study investigated the effect of auditory and tactile keyclick feedback on typing performance in addition to the ever-present visual feedback. We hypothesized 
that tactile feedback could be an additional information channel for improving typing performance when using ten fingers on a QWERTY soft keyboard on a tablet. To test this hypothesis, we developed a prototype with haptic keyclick feedback using a commercial mobile device (Microsoft Surface Pro). We embedded piezoelectric actuators under the touch screen to simulate key-click sensations. Our experiment involved typing tasks under three different feedback conditions: visual feedback, visual with aural feedback, and visual with tactile feedback. We also examined participants' preferences for the three feedback conditions through a questionnaire.

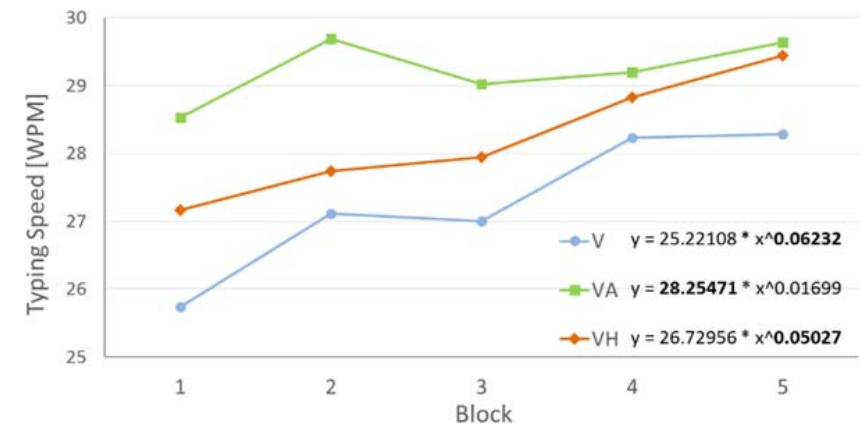

Fig. 4. WPM over the five blocks and fitted power law models with multi-level linear regression. The bold coefficients mean significant at the $95 \%$ confidence level.

Our results did not show significant difference among the three feedback conditions in all the performance metrics tested: typing speed, typing efficiency (keystroke per character) and accuracy (corrected and uncorrected errors). Several factors may have contributed to this result that appears to contradict reports in the literature and our expectations. First, our results might have been affected by learning during the experiment. It is possible that by the end of the experiments, our participants were still getting used to the feedback conditions. We therefore conducted additional data analysis to look at the training effect. The results confirm that there was a training effect across blocks in typing speed for all three conditions during the experiment, shown in Figure 4. The learning effect was significant in $\mathrm{V}$ and VH, but not in VA. These models also show that VH will outperform VA after six blocks whereas $\mathrm{VH}$ and $\mathrm{V}$ will stay in parallel even for a longer run with $\mathrm{VH}$ faster (though not significant). This result suggests that different feedback modalities could provide different improvements on user performance over time, which encourages a future investigation with a longer-term study. Second, the delay between finger press and visual / auditory / tactile feedback may have caused the participants to slow down with their typing and may have buried the potential performance differences among the three feedback conditions.

Although our results are somewhat negative, we believe that this study is still informative for researchers investigating tactile feedback on text entry because it offers different results from prior work. This also calls for a more careful assessment of the conditions under which tactile feedback might enhance user performance, as opposed to taking it for granted that any tactile feedback will help. In this sense, this 
work opens up new opportunities to re-examine the benefits of tactile feedback for touch screen devices. Future work should examine the above-mentioned factors more carefully for a better understanding of the impact of tactile feedback on soft QWERTY keyboards on table devices.

Acknowledgement We thank Darren Edge for providing valuable comments regarding the experimental design, and Magnetro Chen and Zhaoyuan Ma for the control circuit design of our tactile feedback system.

\section{References}

1. Rabin, E. and Gordon, A.M. (2004) "Tactile feedback contributes to consistency of finger movements during typing”. Exp. Brain Res, vol 155, 362-369.

2. Findlater, L., Wobbrock, J., and Wigdor, D. (2011) "Typing on flat glass: examining ten-finger expert typing patterns on touch surfaces". In Proc. CHI 2011, 2453-2462.

3. Brewster, A.A, Chohan, F., and Brown, L. (2007) "Tactile feedback for mobile interactions." In Proc. CHI 2007, 159-162.

4. Hoggan, E., Brewster, S.A., and Johnston, J. (2008) "Investigating the effectiveness of tactile feedback for mobile touch screens", In Proc. CHI 2008, 1573-1582.

5. McAdam, C. and Brewster, S. "Distal tactile feedback for text entry on tabletop computers". In Proc. BCS HCI 2009, 504-511.

6. Jin Ryong Kim and Hong Z. Tan, "A study of touch typing performance with keyclick feedback," Proceedings of the Symposium on Haptic Interfaces for Virtual Environment and Teleoperator Systems, Houston, TX, pp. 227-233, February 23-26, 2014

7. Chen, H.Y., Park, J., Dai, S., and Tan, H.Z. (2011). "Design and evaluation of identifiable key-click signals for mobile devices." IEEE Transactions on Haptics, vol 4, no 4, 229-241.

8. MacKenzie I.S, and Soukoreff, R.W. (2003) "Phrase sets for evaluating text entry techniques." In CHI Extended Abstract 2003, 754-755.

9. Soukoreff, R.W., and MacKenzie, I.S. (2003) "Metrics for text entry research: an evaluation of MSD and KSPC, and a new unified error metric." In Proc. CHI 2003, 113-120.

10. Soukoreff, R.W. and MacKenzie, I.S. (2001) "Measuring errors in text entry tasks: an application of the Levenshtein string distance statistic." In CHI Extended Abstract 2001, 319-320. 